\title{
Concept of mechanocaloric granular material made from shape memory alloy
}

P. Jongchansitto ${ }^{\text {a }}$, T. Yachai ${ }^{\text {a }}$, I. Preechawuttipong ${ }^{\text {a }}$, R. Boufayed ${ }^{\text {b }}$, and X. Balandraud ${ }^{\text {b,* }}$

${ }^{a}$ Chiang Mai University, Faculty of Engineering, Department of Mechanical Engineering, 239 Huay Kaew Rd., Muang District, Chiang Mai 50200, Thailand

${ }^{\mathrm{b}}$ Université Clermont Auvergne, CNRS, SIGMA Clermont, Institut Pascal, F-63000

Clermont-Ferrand, France

*Corresponding author. Tel.: +33 4732880 89. Fax: +33 4732881 00. E-mail address:

xavier.balandraud@sigma-clermont.fr

\begin{abstract}
:
Shape memory alloys (SMAs) are promising mechanocaloric materials for the creation of innovative heating/cooling systems. The present paper proposes a simple concept of "porous" SMA material in the form of a two-dimensional (2D) "granular" system. The basic idea is to place SMA cylinders in parallel and in contact, thus creating a 2D granular material, with fluid circulation in the third dimension (i.e. in the longitudinal direction of the cylinders). The study is dedicated to the validation of the mechanocaloric character of such a material system subjected to confined compression. First, experiments were conducted using superelastic nickel-titanium rods of various diameters, enabling us to create different bidispersity levels for the granular medium. Infrared thermography was used to analyze the temperature changes within the cylinders. Second, air was used as the circulating fluid for the sake of concept validation. Comparison between different levels of bidispersity of the granular system showed that a nearly-monodisperse configuration could be advantageous to optimize the concept.
\end{abstract}

\section{Keywords:}

SMA; Barocaloric effect; Discrete material; Porous material; Infrared thermography 


\section{Introduction}

Shape memory alloys (SMAs) belong to the family of smart and active materials. Superelasticity, one-way and two-way memory effects, as well as strong damping capacities are peculiar mechanical properties of these metallic materials [1-4]. The macroscopic properties of SMAs have their origin in a first-order diffusionless solid-solid phase transition, namely a reversible martensitic transformation triggered by temperature and stress applied to the material. The austenite-to-martensite phase change occurring in SMAs is accompanied by large reversible strains (several \%) and a strong release of latent heat (several $\mathrm{kJ} / \mathrm{kg}$ ), opening perspectives for applications in various engineering domains. Numerous patents involving SMA components have been filed in the last decades. The review by Jani et al. [5] cites more than one hundred state-of-the-art patents in the biomedical, automotive, aerospace and robotics fields. The review by Yuan et al. [6] cites fifty patents only for rotary actuators. In fact, SMAs have been used as mechanical actuators or thermal sensors in a multitude of devices. More recently, another type of application has emerged in the energy domain, relying on the elastocaloric (i.e. under uniaxial mechanical loading) characters of these materials. A few patents have recently been filed in this field (see Refs [7-10]). In particular, their potential as solid-state cooling or heat pumping systems is promising, as they address environmental considerations. Many studies have concerned the analysis of the mechanocaloric properties of SMAs for potential use in such systems. Recently, Zhang et al. (2020) reviewed the properties of both elastocaloric (under uniaxial stress) and barocaloric materials (under hydrostatic pressure) in terms of isothermal entropy change and adiabatic temperature change [11]. Infrared (IR) thermography is often used to measure temperature variations at the surface of SMA samples under mechanical loading [12-18]. Alloys are generally compared in terms of temperature change amplitude or Coefficient of Performance, which measures the energy conversion efficiency from the intrinsic properties of the material [18-20]. Some studies have presented actual physical demonstrators with the ingenious integration of SMA components in contact with fluids or solid parts for heat transfer. SMAs have been employed in the form of foils or plates [21-25], wires [26, 27] and tubes [28], which present topological advantages for efficient heat transfer. It is worth noting that there are a small number of geometries available for SMA components on the market: mainly wires, but also rods, springs, foils, plates and tubes. This is mainly due to the elaboration process of the alloy, which generally requires cold working prior to heat treatment. Numerical optimizations of key geometrical parameters were proposed 
in Refs [29-32]. Potential geometries, including porous architectures, were also discussed in Ref [33].

Porous nickel-titanium-based (Ni-Ti-based) SMAs can be elaborated for applications in biomechanics, such as permanent bone implants [34]. Several manufacturing processes based on powder metallurgy have been applied for this purpose, such as hot isostatic pressing (capsule-free, with gas entrapment, or with space holders), self-propagating high-temperature synthesis, or sintering (spark plasma, microwave, or conventional). Additive manufacturing by selective laser melting has recently opened new possibilities for the production of porous monolithic Ni-Ti-based parts [35, 36]. The issue with these techniques is the control of the size and volume fraction of the pores, as well as of the chemical composition and metallurgical microstructure. The advantages are excellent biocompatibility, high corrosion resistance, low Young's modulus, high strength, as well as good damping and energy-absorbing capacity. Although the resulting three-dimensional intercommunicating porous structure enables fluid transmission (body fluid [37]), these monolithic SMAs have never been devoted to energy applications related to the mechanocaloric effect, to the best of the authors' knowledge.

In this context, the objective of the present study was to build a mechanocaloric "granular" material made from SMA: see Fig. 1-a. The basic idea is to place SMA cylinders in parallel and in contact, thus creating a two-dimensional (2D) granular system, with fluid circulation in the third dimension (i.e. in the longitudinal direction of the cylinders): see Fig. 1-b. The use of straight wires or rods largely available on the market eliminates the need for the complex production of porous monolithic alloys. In addition, strong compression states are possible without buckling thanks to the contact forces between the 2D "particles" under radial loading, i.e. perpendicularly to the axis of the cylinders. The proposed geometrical architecture can be seen as close to the rod-in-tube configuration shown in figure 7(b) of Ref [29], the multi-tube configuration shown in figure 3(b) of Ref [28], or the geometries shown in figure 3(a-d) of Ref [33]. However, the difference is in the granular nature of the proposed material system (i.e. with an interparticle contact force network) and in the direction of fluid circulation. Granular materials exhibit complex behaviors whose macroscopic features are governed by contact forces at a local scale. Under mechanical loading, discrete media are characterized by an inhomogeneous network of interparticle forces. To the best of the authors' knowledge, granular media made from SMA have never been proposed in the literature, and their phase-transforming conditions (if any) are not known. Finally, it must be noted that the goal of the study was not to 
develop a heating/cooling system but to validate the mechanocaloric character of the proposed material system for potential use as both a porous and mechanocaloric medium.

The terms "mechanocaloric granular system", "SMA granular system" or simply "granular system" are used throughout the paper to designate a 2D discrete medium made of SMA cylinders. The paper is organized as follows. Section 2 describes the experimental setup based on IR thermography to observe the thermal response of SMA granular systems subjected to confined compression. Section 3 presents a preliminary finite element (FE) simulation that was performed to estimate an order of magnitude for the external force to be applied on the granular systems to achieve phase transformation. Experimental results are presented and analyzed in Section 4. Finally, Section 5 presents a validation of the general concept proposed in Fig. 1-b using air as the fluid circulating through the SMA granular system.

\section{Materials and methods}

Before validating the concept presented in Fig. 1-b, a test campaign was carried out to analyze the thermal response of different SMA granular systems under confined compression. Figure 2 shows the experimental setup. It involved a rectangular steel frame $26 \mathrm{~mm}$ in width (Fig. 2-a), a uniaxial testing machine to apply a vertical compression load, and an IR camera to measure the temperature changes (Fig. 2-c). Matt black paint was applied to maximize the thermal emissivity of the surfaces observed by the camera (Fig. 2-b). The close environment was covered with thick black fabric to avoid reflections in the IR range during the measurements. A cardboard tunnel (not shown) was also placed between the camera lens and the observed zone in order to minimize any parasitic effects from the thermal environment.

A remark can be made about the type of mechanocaloric effect in the granular systems under test. The macroscopic stress state is biaxial, but the mean pressure on the top/bottom horizontal walls is a priori higher than that on the left/right vertical walls. This is a classic result obtained from simulations by discrete element methods using elastic particles. However, the macroscopic response can be considered as much closer to a barocaloric effect than to an elastocaloric effect. At the particle scale, the effect cannot be really considered as barocaloric for two reasons. First, the radial loading on the particles is not homogeneous: the contacts are 
at most three or four per particle. Secondly, the contact forces are not necessarily normal because of the friction between the cylinders, potentially creating tangential components.

Three diameters were considered for the SMA cylinders: $D_{\mathrm{S}}=2.90 \mathrm{~mm}, D_{\mathrm{M}}=3.30 \mathrm{~mm}$ and $D_{\mathrm{L}}$ $=3.81 \mathrm{~mm}$. Subscript capital letters $\mathrm{S}, \mathrm{M}$ and $\mathrm{L}$ are used throughout the paper to designate "small", "medium" and "large" diameters, respectively. Cylinders of $25 \mathrm{~mm}$ length were cut from 2-meter-long bars of the NiTi-based alloy \#1 supplied by Fort Wayne Metals, Ireland [38]. The cut sections were then lightly polished under water, paying attention not to overheat the material. This alloy was selected because it is superelastic at ambient temperature $\left(T_{\mathrm{amb}}=\right.$ $24.5^{\circ} \mathrm{C} \pm 0.5^{\circ} \mathrm{C}$ for the whole test campaign). Its so-called austenite-finish temperature $A_{\mathrm{f}}$ is equal to $14^{\circ} \mathrm{C}$. As $A_{\mathrm{f}}<T_{\mathrm{amb}}$, the alloy is therefore purely austenite in the stress-free state at ambient temperature. Under uniaxial tensile loading, it features an austenite-to-martensite transition (so-called direct transformation) upon loading and a martensite-to-austenite transition (reverse transformation) upon unloading. The supplier data sheet indicates stress levels in uniaxial tension at $22^{\circ} \mathrm{C}$ of about $450 \mathrm{MPa}$, and $100 \mathrm{MPa}$ for the direct and reverse transformations respectively, i.e. upon loading and unloading respectively [38]. It is worth noting that a huge body of literature is devoted to NiTi-based SMA wires subjected to uniaxial tension. More rarely, experimental studies have been dedicated to their response in bending, torsion and axial compression. The conditions for phase transformation under radial loading (perpendicularly to the cylinder axis) are not established in the literature.

Various granular systems were prepared by mixing the three types of SMA cylinder within the rectangular frame. It is important to note that the choice of numbers and diameters is a critical point. For example, too large a gap between the diameters of small and large particles leads to a lack of stress in small particles if there are not enough of them. On the other hand, too small a difference between small and large diameters leads to a monodisperse configuration, which minimizes the volume available for the fluid and requires higher loading levels. Three configurations are presented in Fig. 3. Two bidisperse cases were set up by mixing cylinders $\mathrm{L}+\mathrm{S}$ and cylinders $\mathrm{M}+\mathrm{S}$. It should be noted that the latter case (Fig. 3-b) corresponds to a diameter ratio $D_{\mathrm{M}} / D_{\mathrm{S}}$ equal to 1.14 . As this value is lower than 1.2 , the macroscopic response is expected to be similar to that of a monodisperse case [39]. This $\mathrm{M}+\mathrm{S}$ granular system is described as nearly-monodisperse in the following. An actual monodisperse case was also created using only the largest diameter (L), see Fig. 3-c. 
Mechanical loading was applied by a Schenck uniaxial machine equipped with a $\pm 250 \mathrm{kN}$ load cell. The force sensor is class 0.5 and was calibrated according to the ISO 7500-1:2018 standard [40]. In compression, it features a force resolution of $0.08 \%$. The loading was displacementcontrolled with a rate of $1 \mathrm{~mm} / \mathrm{s}$ up to a maximum compression force designated $F_{\max }$ in the following. The loading rate was defined after some preliminary tests (not presented here) to ensure significant temperature changes in the granular systems. Let us remark that for the concept illustrated in Fig. 1-b, the loading rate should be a priori slow enough to allow the released heat to be transmitted to the fluid. For the present tests, on the contrary, the loading rate must be high enough to detect significant temperature changes within the cylinders. Systematically, three compression cycles were first applied to ensure the granular system was compacted. A waiting period of 10 minutes was respected before starting each test in order to begin from a steady thermal state. Note that mechanical irreversibility (in particular friction) during cyclic loading leads to the production of mechanical dissipation (also called intrinsic dissipation) tending to increase the magnitude of the heating effect.

A Cedip Jade III-MWIR infrared camera (wavelength range of 3.5-5 $\mu \mathrm{m}, 320 \times 240$ pixels was used to measure the temperature fields on the plane circular surfaces of the cylinders during mechanical loading. Its measurement resolution, referred to as Noise Equivalent Temperature Difference (NETD), is $0.02^{\circ} \mathrm{C}$. The spatial resolution of the thermal measurement, i.e. the pixel size projected onto the observation plane, was equal to $100 \mu \mathrm{m}$. Acquisition frequency was set to $147 \mathrm{~Hz}$, corresponding therefore to a temporal resolution of $6.80 \mathrm{~ms}$. The analysis in the next sections is focused on the temperature changes $\theta$ caused by the thermomechanical couplings occurring in the cylinders. This quantity is obtained by subtracting the initial temperature field captured just before starting the loading. Before showing and analyzing the test results, the next section presents a FE simulation performed in order to assess a suitable order of magnitude for the maximum force $F_{\max }$ to be applied. 


\section{Preliminary finite element simulations}

Discrete-element codes that are available to simulate granular materials do not include SMA superelastic behavior for the particles. It was therefore decided to carry out FE simulations in order to estimate the external force level required to achieve an austenite-to-martensite transformation under confined compression. The monodisperse system (Fig. 3-c) was selected for the calculations as it is the most compact, needing therefore a priori a higher external loading level to activate the phase transformation compared to the bidisperse cases. An Ansys package was employed using Auricchio's model for superelastic behavior [41]. It should be noted that the objective here was not to reproduce a precise experimental configuration. Indeed, it is well known that the local response in granular materials is strongly sensitive to tiny local fluctuations in the input parameters (dimensions, geometries and locations of the particles, bulk material properties, surface properties), which justifies the statistical or macroscopic analyses classically carried out in the literature dedicated to these materials [39, 42-44]. The objective of the present simulation was simply to identify an order of magnitude for the external vertical force to be applied to achieve an austenite-to-martensite transformation. Various force levels were numerically applied until a significant phase transformation in the interparticle contact zones was observed. Figure 4 shows for instance the field of martensite volume fraction $x$ in the monodisperse granular system subjected to a vertical compressive force of $-150 \mathrm{kN}$. In this figure, $x=0$ (blue colour) and $x=1$ (red colour) correspond to pure austenite and pure martensite respectively, whereas intermediate values correspond to a mixing of the two phases. It can be seen in the figure that the austenite-to-martensite transition occurs in the contact zones, as expected due to the stress concentrations. Martensite is more present in the top and bottom rows of cylinders, which can be explained by the fact that these cylinders are subjected to fewer contacts than the others. However, as indicated above, it is tricky to go further in a local analysis from this FE simulation. Nevertheless, the macroscopic information that can be gained for the monodisperse case is that the order of magnitude of the external vertical force creating a significant phase transformation in the contact zones is of about $-150 \mathrm{kN}$. Lower values are expected for the bidisperse cases, because the latter involve fewer contacts (therefore transmitting more contact force for the same external loading). The next section presents the results of the experimental tests. 


\section{Experimental results}

This section presents some results of the experimental test campaign performed on the three granular systems in Fig. 3. The objective was to confirm, thanks to IR thermography, a release of latent heat due a phase transformation under confined compression, and to compare the three configurations in terms of calorific responses. The middle panel of Fig. 5 shows the relationship between the vertical force and the displacement of the actuator for the bidisperse case (Fig. 3a) up to $F_{\max }=-100 \mathrm{kN}$. The first part of the curve is quite linear, which can be explained by the fact that the granular system was initially compacted (no large rigid-body movements of the cylinders). The slope then decreases from a force of about $-90 \mathrm{kN}$, which can be attributed to phase transformation. This can be confirmed by observing the different fields of temperature change $\theta$ that are displayed in the figure. Rapid heating occurred in one of the small cylinders, featuring a temperature rise of more than $12^{\circ} \mathrm{C}$. This value is close to the adiabatic temperature change of $13^{\circ} \mathrm{C}$ measured under axial loading on the same alloy [45]. Considering a typical value of $490 \mathrm{~J} /(\mathrm{kg} \cdot \mathrm{K})$ for the specific heat, this gives a latent heat of about $6 \mathrm{~kJ} / \mathrm{kg}$. This value is of the same order of magnitude of that identified by Gu et al. (2020) in uniaxial tension: latent heat decreased from $11.3 \mathrm{~kJ} / \mathrm{kg}$ to $8.7 \mathrm{~kJ} / \mathrm{kg}$ after a few hundred stress-induced transformation cycles [46]. It is interesting to note that temperature strongly increases in this cylinder along a short force plateau at $-92 \mathrm{kN}$, as it can be observed in the two thermal maps displayed for this force level. This observation can be related to the classical stress plateau accompanying the austenite-to-martensite transformation in the stress-strain curve of superelastic SMA wires in uniaxial tension. Finally, let us note that temperature actually started to increase earlier during loading (see the map for $-70 \mathrm{kN}$ ), revealing an early phase transformation.

Figure 6 shows the results obtained for the nearly-monodisperse case (Fig. 3-b). Compared to the previous configuration, a higher compression force $(-150 \mathrm{kN})$ was required to reach a temperature rise of $12^{\circ} \mathrm{C}$. However, more cylinders appear to be subjected to significant temperature increases (compare with Fig. 5). In particular, an inclined row of several "hot" cylinders is visible in the thermal map. Finally, Figure 7 shows the results obtained for the monodisperse case (Fig. 3-c). A temperature change of barely $4^{\circ} \mathrm{C}$ is achieved in several cylinders for a force of $-170 \mathrm{kN}$. A remark can be made with respect to the numerical comparison in Section 3. In the case of an adiabatic evolution, the martensite volume fraction $x$ should be proportional to the temperature change $\theta$. Indeed, $x$ can be considered proportional 
to the material's change in enthalpy for a complete transition from austenite to martensite. Several reasons explain the large discrepancy between the simulated martensite volume fraction field $x$ in Fig. 4 and the experimental temperature change field $\theta$ in Fig. 7. The experimental response is influenced by: heat diffusion within the cylinders, i.e. local non-adiabaticity; the thermo-elastic coupling effect within the cylinders; and irreversible mechanical phenomena, in particular the interparticle friction which produces intrinsic dissipation [47]. More generally, as already indicated in Section 3, the local response in a discrete medium is strongly sensitive to any tiny variability in the geometry and material at the local scale. As a first conclusion of these experiments, considering the high temperature rises observed, it can reasonably be stated that an austenite-to-martensite transformation was created by the confined compression loading.

A remark can be made about the influence of the loading rate on the mechanical response of the SMA cylinders due to the presence of strong thermomechanical coupling. Indeed, the stressstrain curve in uniaxial tension or compression classically presents a stress plateau during an isothermal test (i.e. at low strain rate). This stress plateau is all the more inclined as the test is anisothermal, thus requiring ever higher stress levels to complete the phase transformation. In other words for our application, the smaller the temperature variation amplitudes in the SMA cylinders during phase transformation (thanks to a strong heat transfer on the surface of the cylinders), the easier the phase transformation is from a mechanical point of view. The use of a fluid circulating between the cylinders thus enables the heat transfer while it is also advantageous from a mechanical point of view. Before employing a fluid (see Section 5), rapid mechanical loading was applied to demonstrate that an austenite-martensite phase transformation does occur during the radial loading of the cylinders.

Two properties are well known in the case of classic elastic particles. First, the more homogeneous the granular system is, the more homogeneous the interparticle force magnitudes are [39]. Second, highest stresses are expected in small particles for polydisperse granular systems; indeed, failure in polydisperse systems generally starts in the smallest particles. The present experimental results for granular systems made of phase-transformation materials appear to be in agreement with these two properties. It should be noted that discrete materials are characterized by wide statistical distributions of contact force magnitudes. For a fixed granular configuration (in terms of the number, shape and size of particles), the spatial distribution of the contacts varies greatly, depending on the positions of the particles. Tiny variations in the shapes or dimensions of the particles, as well as in the boundary conditions, 
also strongly impact the distribution of contact intensities. The following trends can be deduced from the test campaign (see Fig. 8):

- the higher the level of size dispersity, the higher the amplitude of the local heat release (which is good for the targeted application in Fig. 1-b) but the stronger the localization of the phase transformation in the granular system (which is bad for the application);

- the lower the level of size dispersity, the higher the number of cylinders involved by phase transformation (which is good for the application), but the higher the external force level required (which is bad for the application).

The nearly-monodisperse system in Fig. 3-b appears to be a compromise between the two other configurations for the targeted application. The present experimental study has shown that it is possible to consider an optimization of the granular medium. Simulations by discrete element methods integrating superelastic SMA behavior at the contacts could help in the future to optimize the numbers, diameters and locations of the cylinders. Based on these test results, the next section presents a validation of the concept illustrated in Fig. 1-b.

\section{Validation of the concept}

The concept of a "porous" mechanocaloric granular material made from SMA cylinders through which a fluid flows was tested using air for the sake of simplicity. Figure 9-a shows a schematic view of the experimental setup. Air flow through the granular system was created by means of a tunnel and a fan placed at the exit of the granular system. To limit air leaks, duct tape was applied to seal the top of the granular system in the frame. A type-K thermocouple was used to measure the output temperature $T_{\text {air }}$, see Fig. 9-b. The recording frequency chosen for temperature measurement was $10 \mathrm{~Hz}$ from a TC-08 USB thermocouple data logger (Pico Technology). The base measurement resolution of the thermocouple is $0.025^{\circ} \mathrm{C}$. The "average" sample mode available in PicoLog 6 software [48] enabled us to improve this measurement resolution: the standard deviation of the noise during a steady thermal regime (fan rotating without cyclic mechanical loading) was measured at less than $0.007^{\circ} \mathrm{C}$ at $10 \mathrm{~Hz}$ thanks to this time filter. The location of the thermocouple in the tunnel was defined from numerical simulations using SOLIDWORKS Flow Simulation software (2020). Various revolutions per minute (rpm) values were applied to the fan, ranging from $220 \mathrm{rpm}$ to $3200 \mathrm{rpm}$. Various temperature distributions with temperature variations were also applied to the cylinders. For 
instance, Figure 10-a shows the results for a fan rotation speed of $3200 \mathrm{rpm}$ with a maximum temperature change of $4^{\circ} \mathrm{C}$ in some cylinders. As expected, the air temperature drops rapidly outside of the granular material. A compromise was sought to find a fixed location for the thermocouple for fan rotations in the range [220 rpm; $3200 \mathrm{rpm}$ ] so that the maximum homogenized air temperature is captured. A distance of $15 \mathrm{~mm}$ from the granular material was found. Figures 10-b and -c show for instance the temperature fields in the thermocouple's plane for $220 \mathrm{rpm}$ to $1500 \mathrm{rpm}$ respectively.

Figure 9-c shows a nearly-monodisperse system placed in the steel frame. It can be noted that the frame geometry was slightly modified compared to that used for the preliminary tests (compare with Fig. 2): in particular, fillets were manufactured to limit stress concentrations; furthermore, two larger steel cylinders were placed at the top left and right corners of the granular system to prevent the SMA cylinders from being forced out during compression. For all the tests, three preliminary compression cycles were applied to ensure that the granular system was compacted. The fan was turned on ten minutes before the beginning of each test in order to start from a steady thermal state. The ambient temperature $T_{\mathrm{amb}}$ was defined as the temperature measured by the thermocouple just before starting mechanical loading. Sinusoidal mechanical loading at frequency $f_{\mathrm{L}}$ was applied to the granular systems while air continued to circulate. For all the tests, the force ratio between the minimum value $F_{\min }$ and maximum value $F_{\max }$ was set to a positive value $\left(F_{\min } / F_{\max }=0.1\right)$ to avoid loss of contact during force oscillation. Analysis focused on the temperature change $\theta_{\text {air }}$ measured by the thermocouple over time $t$ with respect to the ambient temperature: $\theta_{\mathrm{air}}(t)=T_{\mathrm{air}}(t)-T_{\mathrm{amb}}$.

Figure 11 shows the time variation in air temperature change $\theta_{\text {air }}$ at the exit of the nearlymonodisperse SMA granular system for $F_{\max }=-180 \mathrm{kN}$ and $f_{\mathrm{L}}=0.083 \mathrm{~Hz}$, comparing various fan rotation speeds: $220,1200,1700,2200,2500,2800$, and $3200 \mathrm{rpm}$. The first observation is that the magnitude of $\theta_{\text {air }}$ is low compared to the temperature changes $\theta$ measured in the SMA cylinders in Section 4. This was expected because of the fluid used. The order of magnitude is the same as in the experiments by Michaelis et al. in Ref [26], also using an air flow generated by fans around a SMA wire in uniaxial tension. However, two points reveal a heat transfer from the "porous" mechanocaloric material to the circulating fluid. Firstly, a cyclic oscillation of the air temperature is observed at the same frequency as the mechanical loading, whatever the fan rotation speed. Secondly, the temperature started to increase at the first loading stage, in 
agreement with an exothermic austenite-to-martensite transformation upon loading. Finally, let us note that our choice of large diameters and air as the circulating fluid led to energy performances that are lower than those of the devices presented in the literature. However, a significant result of the present study is to highlight the relevance of the nearly-monodisperse configuration, which is a form of compromise between the advantages and disadvantages of the monodisperse and the bidisperse configurations.

Two additional observations can be made for Fig. 11 concerning the amplitude and the mean value of the temperature fluctuation over the cycles: the mean temperature decreases over the cycles but stabilizes above ambient temperature, which can explained by a heat leakage in the metallic frame; and the amplitude of temperature oscillation increases with the rotation speed of the fan and stabilizes from $2800 \mathrm{rpm}$. Other loading frequencies and levels were applied, confirming these tendencies. The two other granular systems were also tested. For example, Figure 12 shows the time variation in air temperature change for the three granular configurations (bidisperse, nearly-monodisperse and monodisperse) for $F_{\max }=-180 \mathrm{kN}$ and a fan rotation speed set to $3200 \mathrm{rpm}$. The loading frequency $f_{\mathrm{L}}$ is equal to $0.083 \mathrm{~Hz}$ for the bidisperse and nearly-monodisperse cases, and three times greater $(0.250 \mathrm{~Hz})$ for the monodisperse case. The void ratios in the three granular systems were almost the same (19\%, $23 \%$ and $22 \%$ - excluding the zone around the two large steel cylinders in the calculation - for the bidisperse, nearly-monodisperse and monodisperse cases respectively), contrary to the volume-to-surface ratio, which decreases with the size dispersity level: $0.84 \mathrm{~mm}, 0.89 \mathrm{~mm}$ and $0.95 \mathrm{~mm}$ respectively. Three comments can be made about the graph. First, temperature oscillation at the loading frequency is confirmed. Second, the monodisperse case features the lowest temperature changes, despite the higher loading frequency and the higher volume-tosurface ratio. Third, the nearly-monodisperse configuration features the highest temperature changes, making it a compromise between the other two cases, in agreement with the global trends indicated in Section 4. 


\section{Conclusion}

SMAs are mechanocaloric materials that have opened perspectives for the creation of new heating/cooling systems. However, the reduced number of geometries available for SMA components limits the architecture possibilities of such devices. In particular, manufacturing monolithic porous SMAs remains complicated, and is dedicated in practice to other applications in biomechanics. The present paper proposes a simple concept of "porous" material in the form of a 2D "granular" system, placing SMA cylinders in parallel and in contact, with fluid circulation allowed in the third dimension, i.e. in the longitudinal direction of the cylinders. The concept was tested in two steps. First, an IR camera was used to confirm the phase-transforming character of the discrete material system under confined compression. It was observed that the granular nature leads by construction to a heterogeneous phase transformation but avoids buckling. Second, the concept was validated using air as the circulating fluid. A comparison between different levels of bidispersity in the granular system showed that a nearlymonodisperse configuration could be advantageous to optimize the concept. The advantages of the proposed 2D granular SMA system are:

- it is simple and cheap compared to monolithic porous SMAs. Indeed, SMA are widely available on the market in the form of straight wires and rods;

- buckling is prevented by the internal contact force network;

- fluid flow is not hindered by the mechanical loading system, whose direction is perpendicular to it;

- the concept can easily accommodate size reduction. SMA wires are available on the market starting at diameters of a few tens of micrometers.

Extrapolating from the present study, the next steps will be the use of a heat-transfer liquid and a dedicated fluid circuit. SMA tubes could also be used to increase the heat transfer area.

\section{Acknowledgements}

The authors gratefully acknowledge the Ministère de l'Europe et des Affaires Etrangères (MEAE) and the Ministère de l'Enseignement supérieur, de la Recherche et de l'Innovation (MESRI) in France, as well as the Office of the Higher Education Commission (OHEC) of the Ministry of Education in Thailand. The authors also gratefully thank the French Embassy in Thailand and Campus France for their support during this research (PHC SIAM 2018, Project 
40710SE). P. Jongchansitto gratefully thanks the CMU Junior Research Fellowship Program. Finally, the authors gratefully thank Mr. Kunanon Jongchansitto, Chiang Mai University, for the preliminary analysis of the thermal images by infrared thermography.

\section{References}

[1] Otsuka K, Wayman CM (1999) Shape Memory Materials. Cambridge University Press, Cambridge, UK.

[2] Lagoudas DC (2010) Shape Memory Alloys: Modeling and Engineering Applications. Springer Science, New York, USA.

[3] Lexcellent C (2013) Shape-Memory Alloys Handbook. John Wiley \& Sons, Hoboken, NJ, USA.

[4] Ziółkowski A (2015) Pseudoelasticity of Shape Memory Alloys: Theory and Experimental Studies. Butterworth-Heinemann.

[5] Jani JM, Leary M, Subic A, Gibson MA. A review of shape memory alloy research, applications and opportunities. Mater Design 2014;56:1078-113. https://doi.org/10.1016/j.matdes.2013.11.084

[6] Yuan H, Fauroux JC, Chapelle F, Balandraud X. A review of rotary actuators based on shape memory alloys. J Intell Mater Syst Struct 2017;28:1863-85. https://doi.org/10.1177/1045389X16682848

[7] Hou H, Takeuchi I, Staruch M, Finkel P. Systems and methods for cooling using a composite elastocaloric device. Patent US2020096240 (A1) - 2020-03-26; 2020.

[8] Sharar DJ, Hanrahan BM. Continuous bending-mode elastocaloric cooling/heating flow loop. Patent US2020088449 (A1) - 2020-03-19; 2020.

[9] Schroeder MG. Method for operating and elasto-caloric heat pump with variable prestrain. Patent US2019323742 (A1) - 2019-10-24; 2019.

[10] Schroeder MG, Benedict MA. Elasto-caloric heat pump system. Patent US2019178536 (A1) - 2019-06-13; 2019.

[11] Zhang JR, Xu YX, An SH, Sun Y, Li XD, Li YC. Giant mechanocaloric materials for solid-state cooling*. Chin Phys B 2020;29:076202. https://doi.org/10.1088/16741056/ab8a40

[12] Ianniciello L, Romanini M, Manosa L, Planes A, Engelbrecht K, Vives E. Tracking the dynamics of power sources and sinks during the martensitic transformation of a $\mathrm{Cu}-\mathrm{Al}-\mathrm{Ni}$ single crystal. Appl Phys Lett 2020;116:183901. https://doi.org/10.1063/5.0006859 
[13] Tusek J, Engelbrecht K, Manosa L, Vives E, Pryds N. Understanding the thermodynamic properties of the elastocaloric effect through experimentation and modelling. Shape Mem Superelasticity 2016;2:317-29. https://doi.org/10.1007/s40830-016-0094-8

[14] Li Y, Sun W, Zhao DW, Xu H, Liu J. An 8 K elastocaloric temperature change induced by $1.3 \%$ transformation strain in Ni44Mn45-xSn11Cux alloys. Scripta Mater 2017;130:278-282. https://doi.org/10.1016/j.scriptamat.2016.12.014

[15] Cui J, Wu YM, Muehlbauer J, Hwang YH, Radermacher R, Fackler S, Wuttig M, Takeuchi I. Demonstration of high efficiency elastocaloric cooling with large Delta T using NiTi wires. Appl Phys Lett 2012;101:073904. https://doi.org/10.1063/1.4746257

[16] Welsch F, Ullrich J, Ossmer H, Schmidt M, Kohl M, Chluba C, Quandt E, Schutze A, Seelecke S. Numerical simulation and experimental investigation of the elastocaloric cooling effect in sputter-deposited TiNiCuCo thin films. Continuum Mech Therm 2018;30:53-68. https://doi.org/10.1007/s00161-017-0582-x

[17] Tusek J, Zerovnik A, Cebron M, Brojan M, Zuzek B, Engelbrecht K, Cadelli A. Elastocaloric effect vs fatigue life: exploring the durability limits of Ni-Ti plates under prestrain conditions for elastocaloric cooling. Acta Mater 2018;150:295-307. https://doi.org/10.1016/j.actamat.2018.03.032

[18] Qian SX, Geng YL, Wang Y, Ling JZ, Hwang YH, Radermacher R, Takeuchi I, Cui J. A review of elastocaloric cooling: Materials, cycles and, system integrations. Int J Refrig 2016;64:1-19. https://doi.org/10.1016/j.ijrefrig.2015.12.001

[19] Moya X, Kar-Narayan S, Mathur ND. Caloric materials near ferroic phase transitions. Nat Mater 2014;13:439-50. https://doi.org/10.1038/nmat3951

[20] Tusek J, Engelbrecht K, Millan-Solsona R, Manosa L, Vives E, Mikkelsen LP, Pryds N. The elastocaloric effect: A way to cool efficiently. Adv Energy Mater 2015;5:1500361. https://doi.org/10.1002/aenm.201500361

[21] Tusek J, Engelbrecht K, Eriksen D, Dall'Olio S, Tusek J, Pryds N. A regenerative elastocaloric heat pump. Nat Energy 2016;1:1-5. https://doi.org/10.1038/nenergy.2016.134

[22] Bruederlin F, Ossmer H, Wendler F, Miyazaki S, Kohl M. SMA foil-based elastocaloric cooling: from material behavior to device engineering. J Phys D App Phys 2017;50:424003. https://doi.org/10.1088/1361-6463/aa87a2

[23] Ossmer H, Wendler F, Gueltig M, Lambrecht F, Miyazaki S, Kohl M. Energy-efficient miniature-scale heat pumping based on shape memory alloys. Smart Mater Struct 2017;25:085037. https://doi.org/10.1088/0964-1726/25/8/085037 
[24] Ossmer H, Miyazaki S, Kohl M. Elastocaloric heat pumping using a shape memory alloy foil device. In: IEEE (editors) 2015 Transducers - 18th International Conference on SolidState Sensors, Actuators and Microsystems. Anchorage, AK, June 21-25, 2015. pp. 72629. https://doi.org/10.1109/TRANSDUCERS.2015.7181026

[25] Schmidt M, Schutze A, Seelecke S. Scientific test setup for investigation of shape memory alloy based elastocaloric cooling processes. Int J Refrig 2015;54:88-97. https://doi.org/10.1016/j.ijrefrig.2015.03.001

[26] Michaelis N, Welsch F, Kirsch SM, Schmidt M, Seelecke S, Schutze A. Experimental parameter identification for elastocaloric air cooling. Int J Refrig 2019;100:167-74. https://doi.org/10.1016/j.ijrefrig.2019.01.006

[27] Snodgrass R, Erickson D. A multistage elastocaloric refrigerator and heat pump with 28 K temperature span. Sci Rep 2019;9:18532. https://doi.org/10.1038/s41598-019-54411-8 [28] Hou H, Cui J, Qian S, Catalini D, Hwang Y, Radermacher R, Takeuchi I. Overcoming fatigue through compression for advanced elastocaloric cooling. MRS Bull 2018;43:28590. https://doi.org/10.1557/mrs.2018.70

[29] Qian SX, Alabdulkarem A, Ling JZ, Muehlbauer J, Hwang YH, Radermacher R, Takeuchi I. Performance enhancement of a compressive thermoelastic cooling system using multi-objective optimization and novel designs. Int J Refrig 2015;57:62-76. https://doi.org/10.1016/j.ijrefrig.2015.04.012

[30] Qian SX, Yuan LF, Yu JL, Yan G. Numerical modeling of an active elastocaloric regenerator refrigerator with phase transformation kinetics and the matching principle for materials selection. Energy 2017;141:744-56. https://doi.org/10.1016/j.energy.2017.09.116

[31] Tan JM, Wang Y, Xu SJ, Liu HC, Qian SX. Thermodynamic cycle analysis of heat driven elastocaloric cooling system. Energy 2020;197:117261. https://doi.org/10.1016/j.energy.2020.117261

[32] Luo D, Feng YS, Verma P. Modeling and analysis of an integrated solid state elastocaloric heat pumping system. Energy 2017;130:500-14. https://doi.org/10.1016/j.energy.2017.05.008

[33] Kabirifar P, Zerovnik A, Ahcin Z, Porenta L, Brojan M, Tusek J. Elastocaloric cooling: State-of-the-art and future challenges in designing regenerative elastocaloric devices. Strojniski Vestnik - J Mech Eng 2019;65:615-30. https://doi.org/10.5545/svjme.2019.6369

[34] Bansiddhi A, Sargeant TD, Stupp SI, Dunand DC. Porous NiTi for bone implants: A review. Acta Biomaterialia 2008;4:773-82. https://doi.org/10.1016/j.actbio.2008.02.009 
[35] Wang XJ, Xu SQ, Zhou SW, Xu W, Leary M, Choong P, Qian M, Brandt M, Xie YM. Topological design and additive manufacturing of porous metals for bone scaffolds and orthopaedic implants: A review. Biomaterials 2016;83:127-41. https://doi.org/10.1016/j.biomaterials.2016.01.012

[36] Elahinia M, Moghaddam NS, Andani MT, Amerinatanzi A, Bimber BA, Hamilton RF. Fabrication of NiTi through additive manufacturing: A review. Prog Mater Sci 2016;83:630-63. https://doi.org/10.1016/j.pmatsci.2016.08.001

[37] Stergioudi F, Vogiatzis CA, Pavlidou E, Skolianos S, Michailidis N. Corrosion resistance of porous NiTi biomedical alloy in simulated body fluids. Smart Mater Struct 2016;25:095024. https://doi.org/10.1088/0964-1726/25/9/095024

[38] Fort Wayne Metal, 2020. https://www.fwmetals.com/materials/nitinol/rod/

[39] Jongchansitto P, Preechawuttipong I, Balandraud X, Grédiac M. Numerical investigation of the influence of particle size and particle number ratios on texture and force transmission in binary granular composites. Powder Technol 2017;308:324-33. https://doi.org/10.1016/j.powtec.2016.11.023

[40] ISO 7500-1:2018 standard. Metallic materials - Calibration and verification of static uniaxial testing machines - Part 1: Tension/compression testing machines - Calibration and verification of the force-measuring system. 2018. https://www.iso.org/standard/72572.html

[41] Auricchio, F. A robust integration-algorithm for a finite-strain shape-memory-alloy. Int J Plasticity 2001;17:971-90. https://doi.org/10.1016/S0749-6419(00)00050-4

[42] Radjai F, Jean M, Moreau JJ, Roux S. Force distributions in dense two-dimensional granular systems. Phys Rev Lett. 1996;77:274-7. https://doi.org/10.1103/PhysRevLett.77.274

[43] Radjai F, Roux JN, Daouadji A (2017) Modeling granular materials: century-long research across scales. J Eng Mech 143:04017002. https://doi.org/10.1061/(ASCE)EM.1943-7889.0001196

[44] Cantor D, Cardenas-Barrantes M, Preechawuttipong I, Renouf M, Azema E. Compaction model for highly deformable particle assemblies. Phys Rev Lett 2020;124:208003. https://doi.org/10.1103/PhysRevLett.124.208003

[45] Jury A, Balandraud X, Heller L, Šittner P, Karlik M. Reconstruction of heat sources induced in superelastically loaded Ni-Ti wire by localized deformation processes. Exp Mech. 2020;in press. https://doi.org/10.1007/s11340-020-00648-8 
[46] Gu XJ, Zhang YH, You YJ, Ju XF, Zhu JH, Moumni Z, Zhang WH. Evolution of transformation characteristics of shape memory alloys during cyclic loading: transformation temperature hysteresis and residual martensite. Smart Mater Struct 2020;29:095011. https://doi.org/10.1088/1361-665X/ab9f10

[47] Jongchansitto P, Balandraud X, Preechawuttipong I, Le Cam JB, Garnier P.

Thermoelastic couplings and interparticle friction evidenced by infrared thermography in granular materials. Exp Mech 2018;58:1469-78. https://doi.org/10.1007/s11340-018-04303

[48] PicoLog Data logging software, User's Guide, p. 31, Pico Technology Ltd. 2020. https://www.picotech.com/download/manuals/PicoLogUsersGuide.pdf 
a)
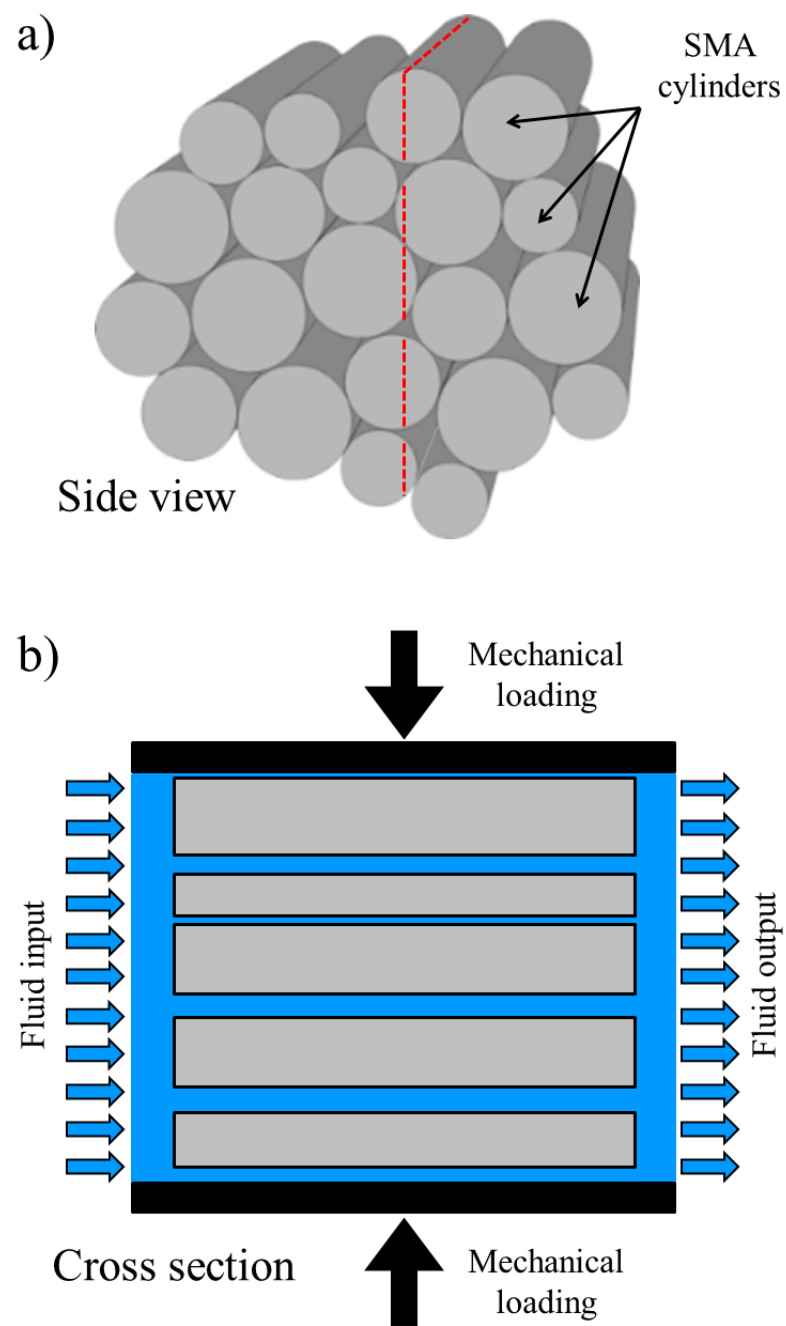

Fig. 1 Concept of elastocaloric granular material made from shape-memory alloy (SMA) rods:

a) cylinders placed in parallel and in contact, forming a two-dimensional granular system; b) fluid flowing through the SMA granular system subjected to radial mechanical loading. 

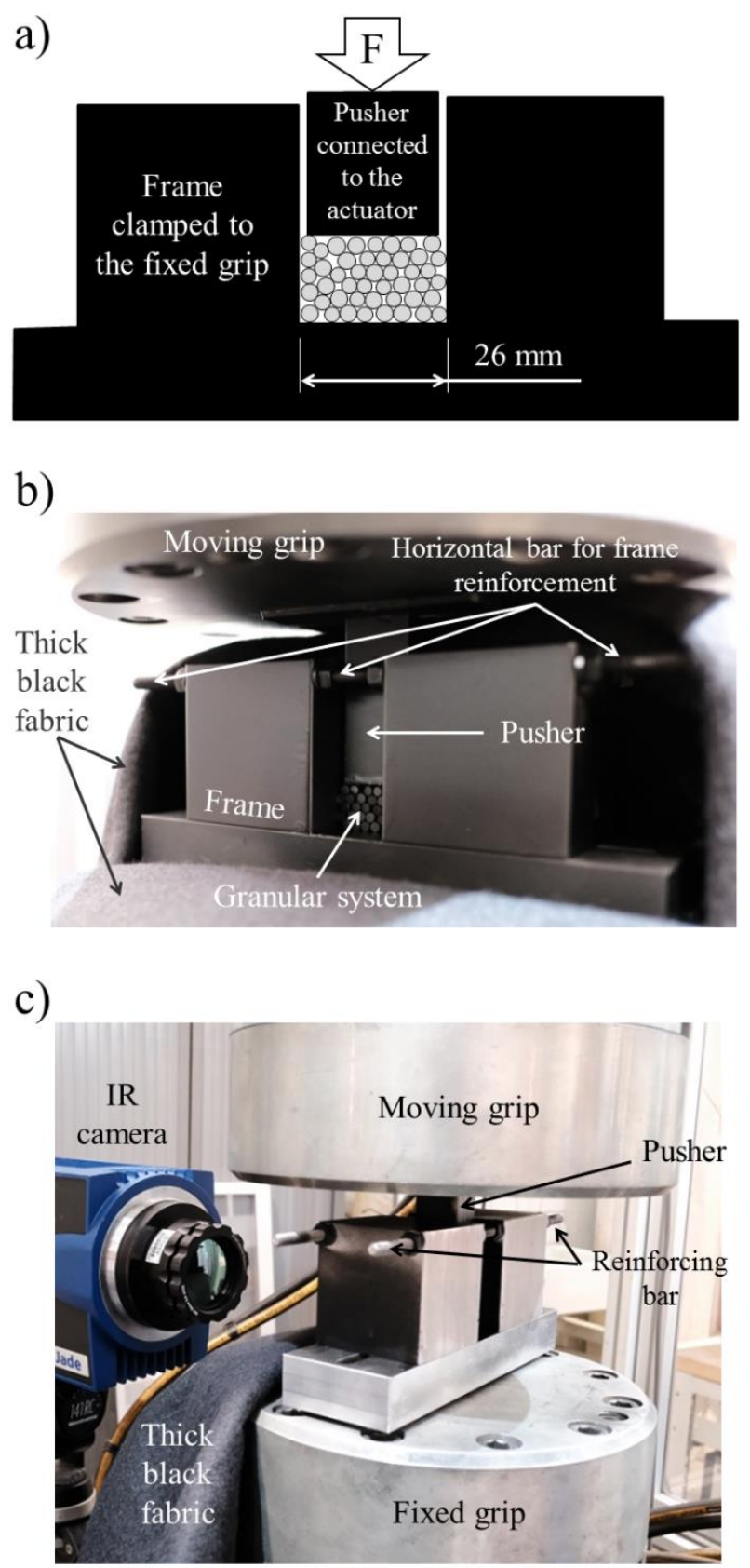

Fig. 2 Experimental setup to demonstrate the mechanocaloric character of the discrete materials under study: a) schematic view of a rectangular frame containing a granular system subjected to confined compression; b) device placed in a uniaxial testing machine; c) view of the experiment instrumented by infrared (IR) camera. 


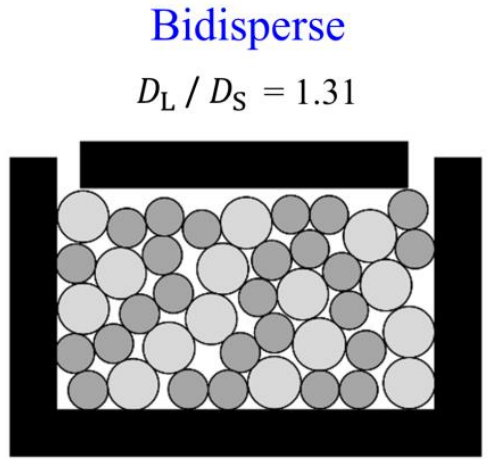

a)
Nearly-monodisperse

$$
D_{\mathrm{M}} / D_{\mathrm{S}}=1.14
$$

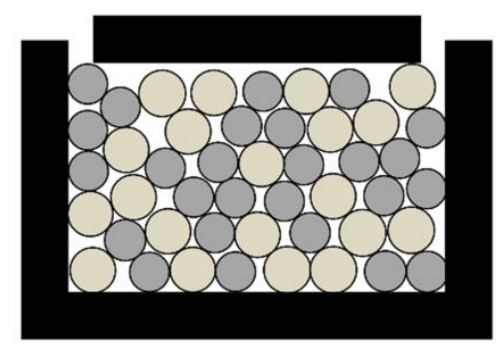

b)
Monodisperse

Only $D_{\mathrm{L}}$

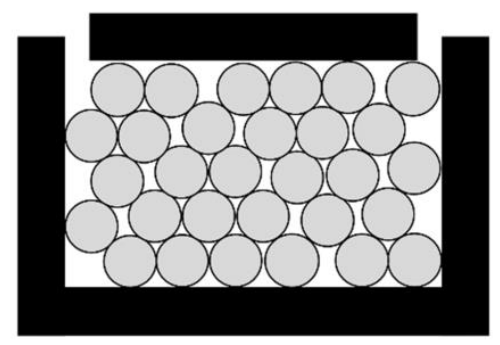

c)

Fig. 3 Schematic view of three SMA granular systems: a) bidisperse configuration; b) nearlymonodisperse configuration; c) monodisperse configuration. Subscript letters S, M and L are used for the small, medium and large cylinder diameters, respectively. 


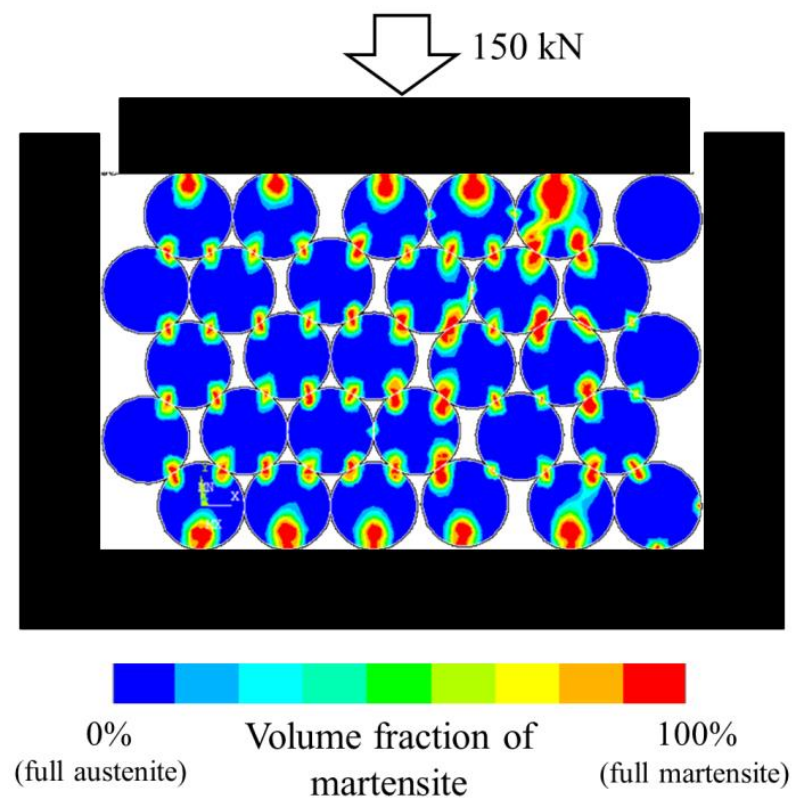

Fig. 4 Field of martensite volume fraction obtained by finite element simulation for the monodisperse SMA granular system in Fig. 3-c. 

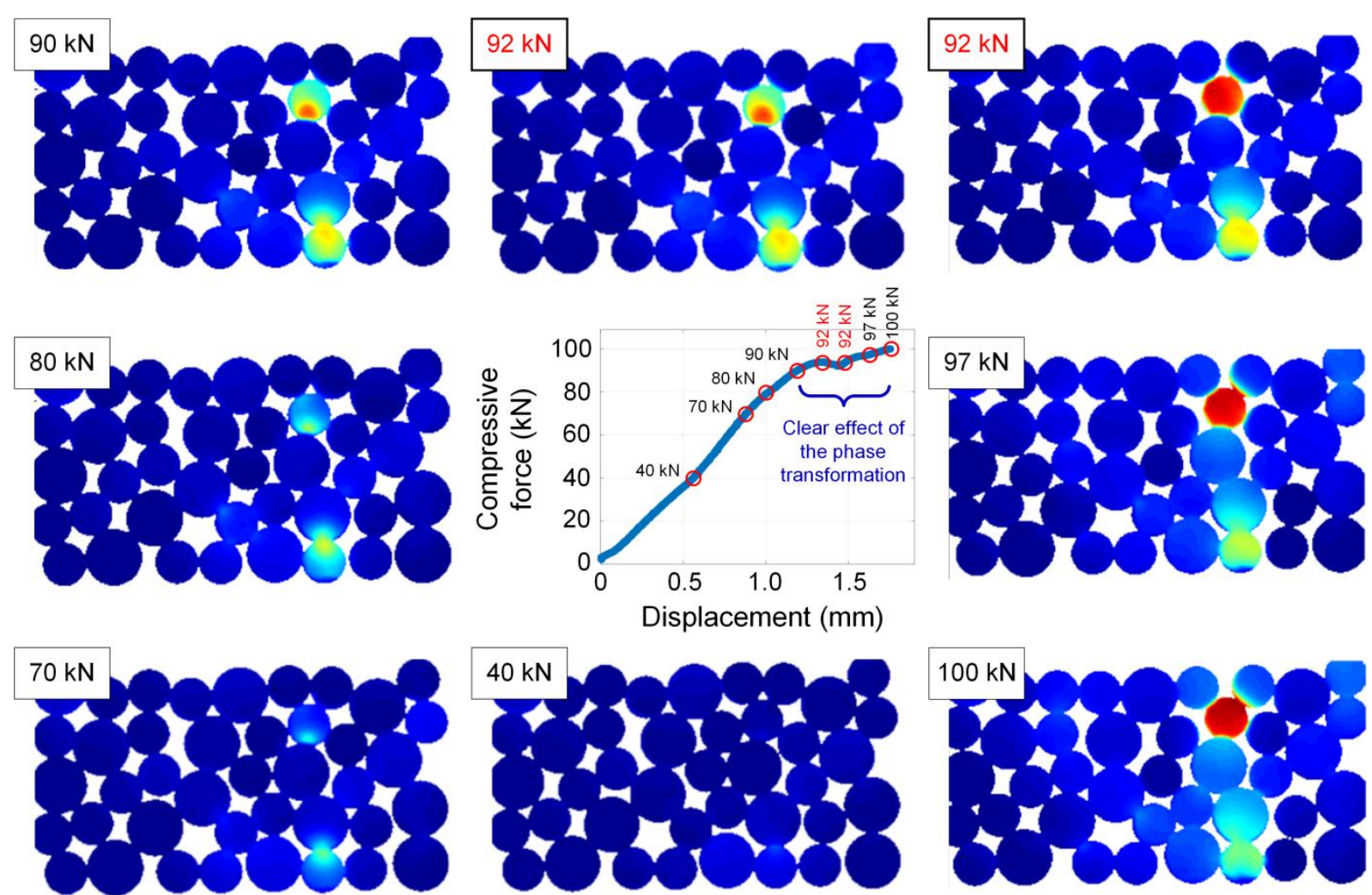

Temperature change $\left({ }^{\circ} \mathrm{C}\right)$

Fig. 5 Results for the bidisperse SMA granular system in Fig. 3-a for a maximum vertical force $F_{\max }$ of $-100 \mathrm{kN}$. 


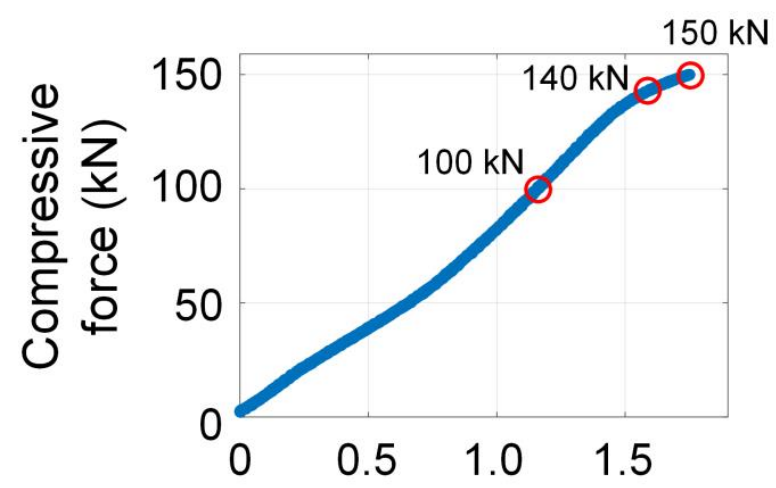

Displacement (mm)
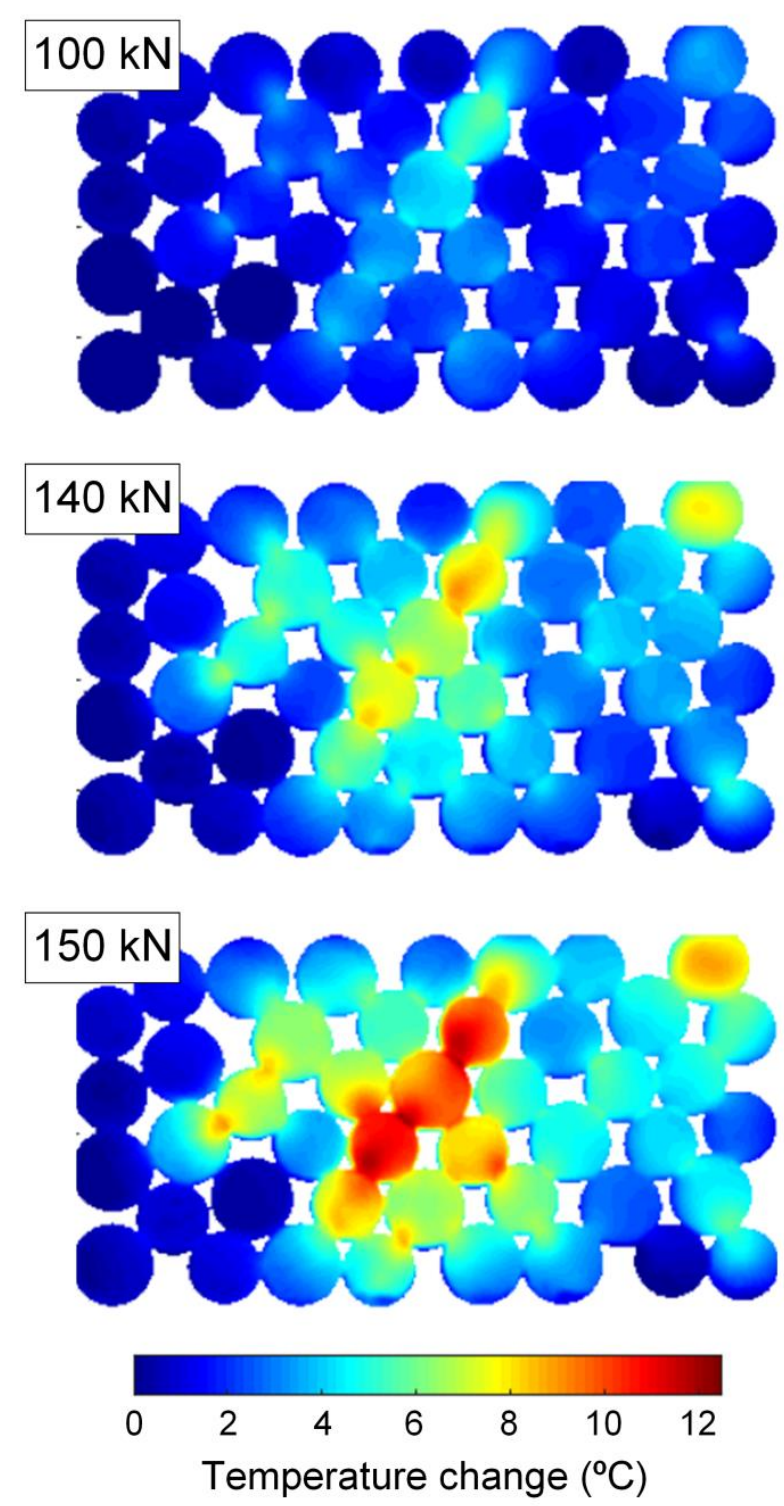

Fig. 6 Results for the nearly-monodisperse SMA granular system in Fig. 3-b for a maximum vertical force $F_{\max }$ of $-150 \mathrm{kN}$. 

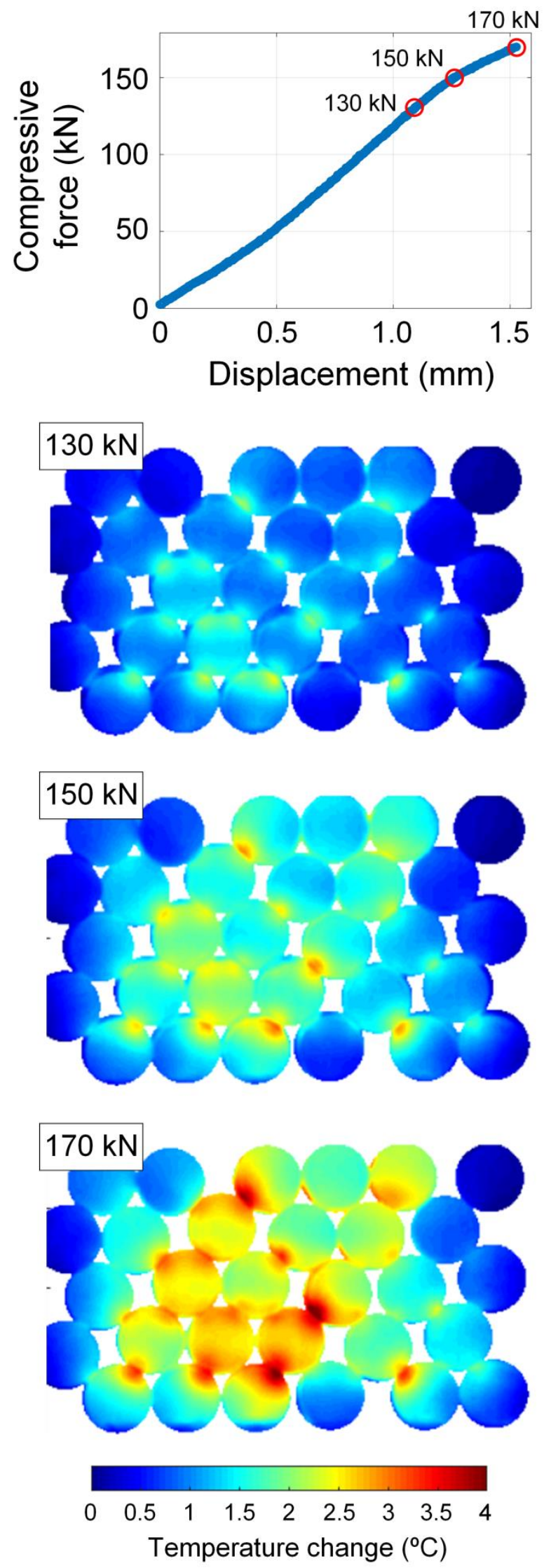

Fig. 7 Results for the monodisperse SMA granular system in Fig. 3-c for a maximum vertical force $F_{\text {max }}$ of $-170 \mathrm{kN}$. 


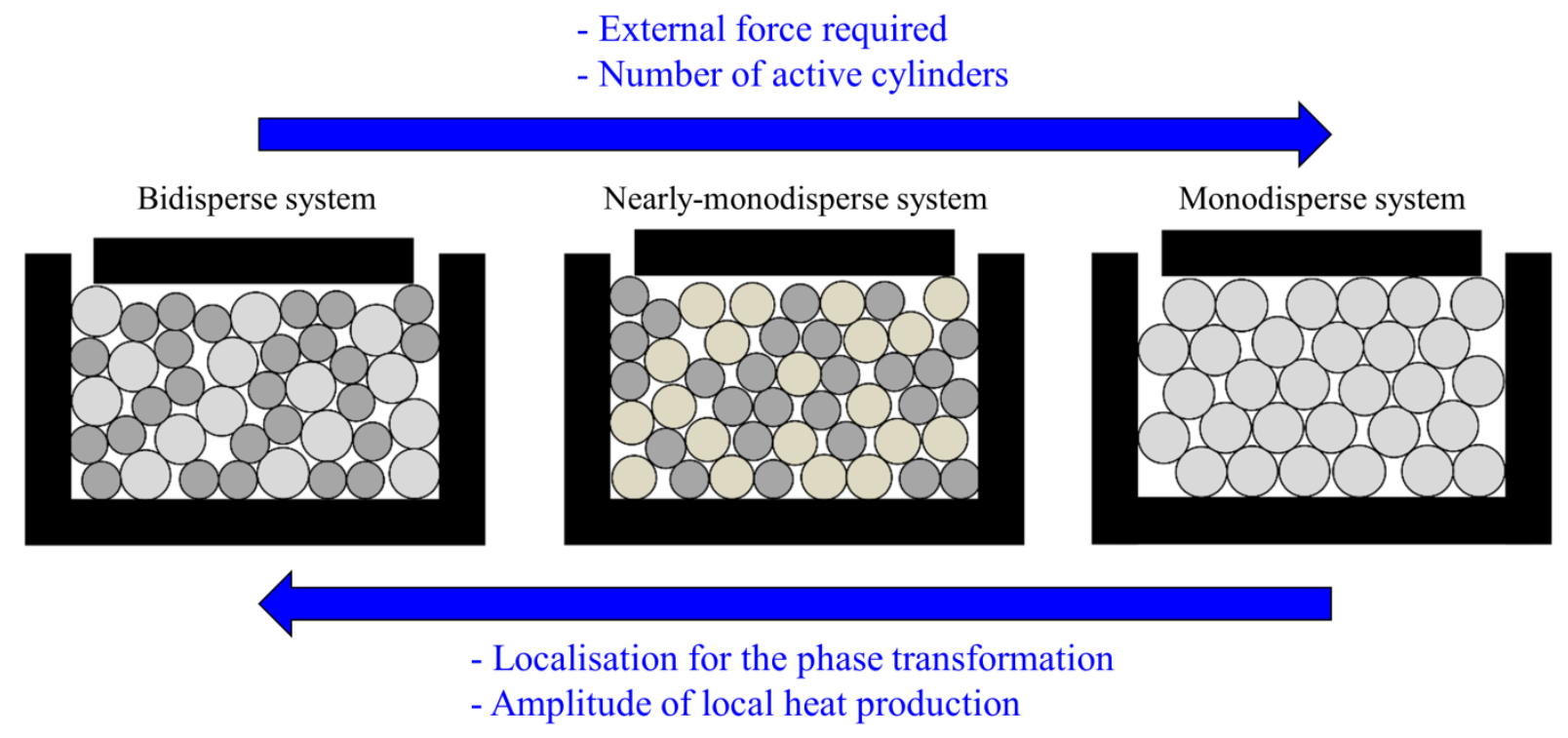

Fig. 8 General trends from experimental results. 

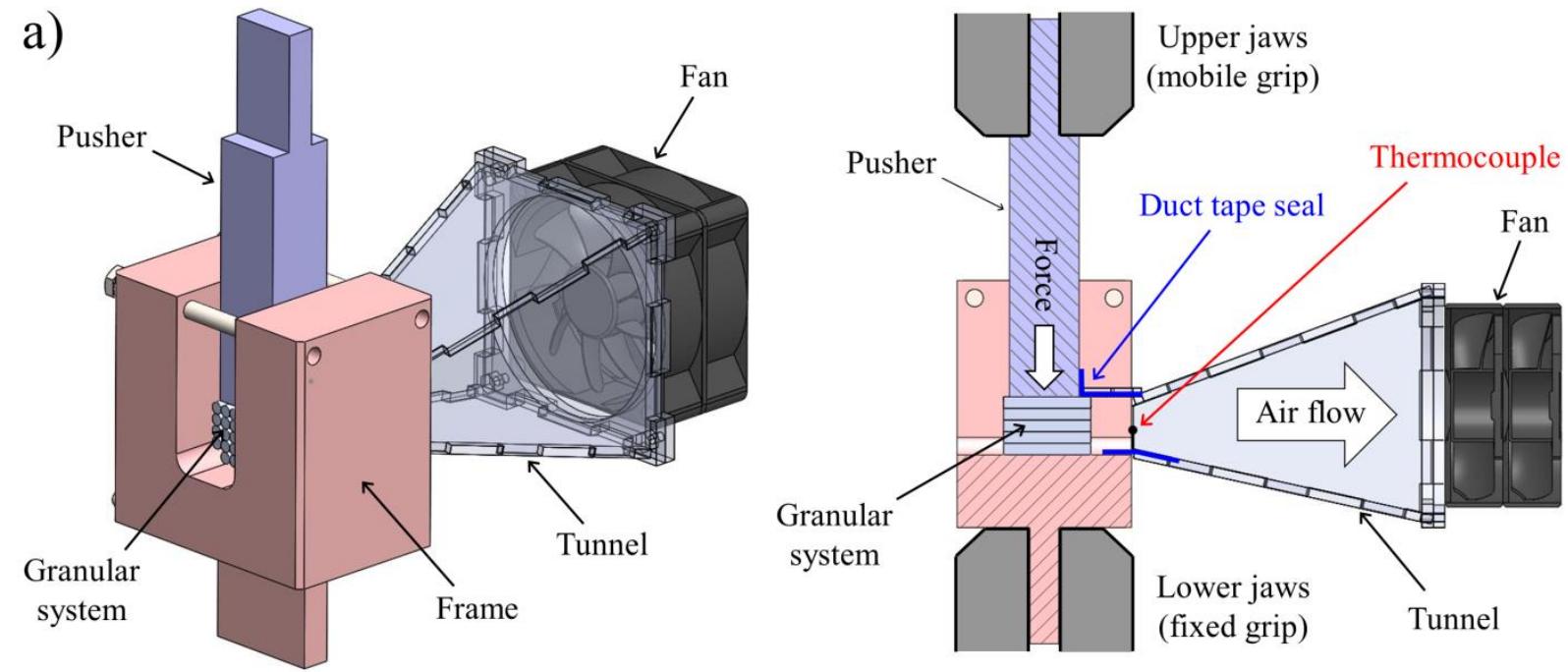

b)

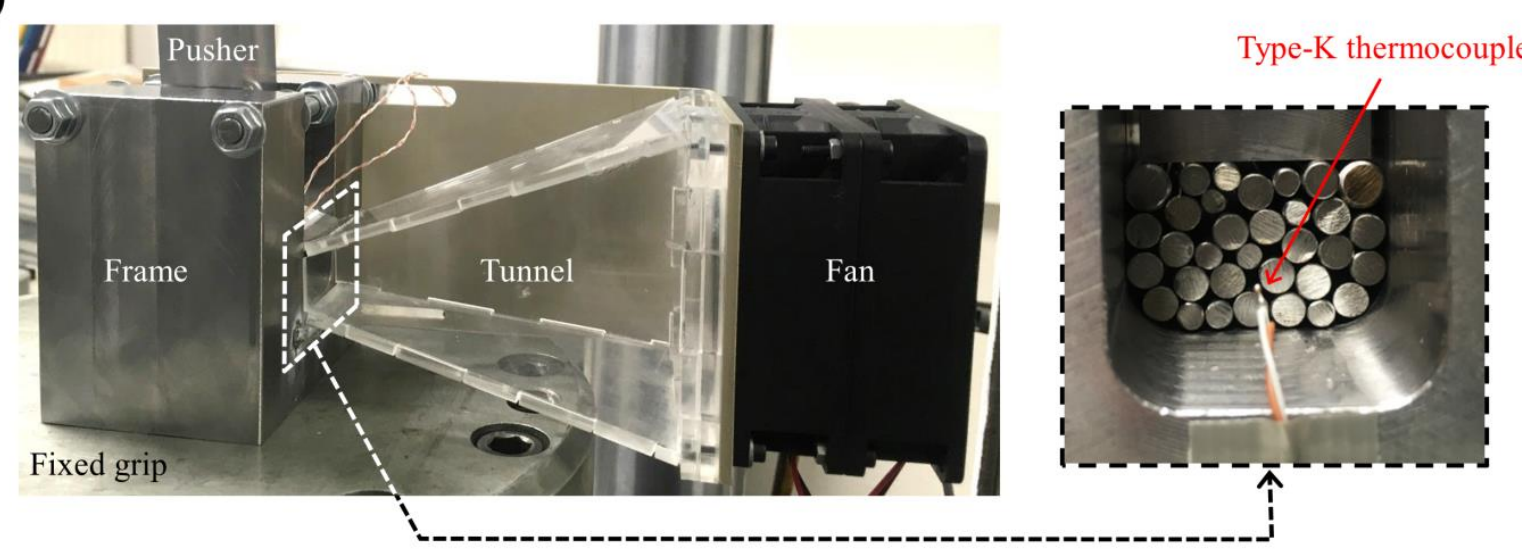

c)

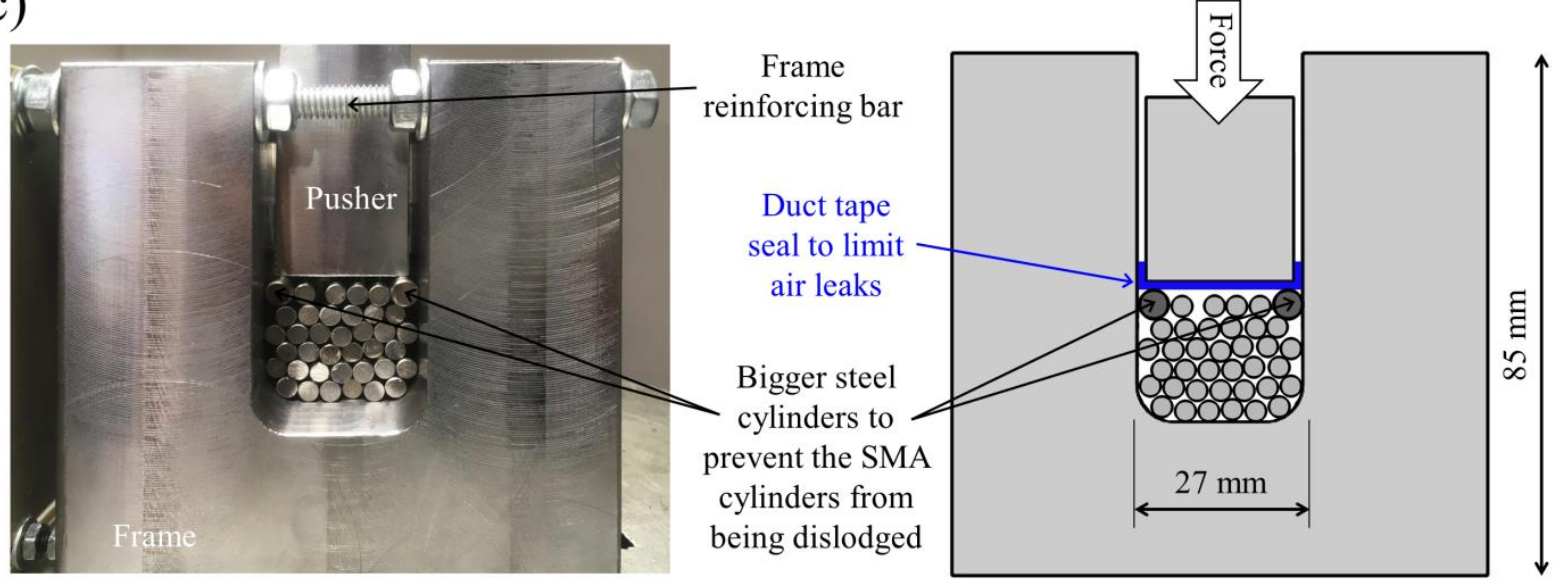

Fig. 9 Experimental setup to validate the general concept presented in Fig. 1-b: a) schematic view of the device; b) photos of the device; c) details. 


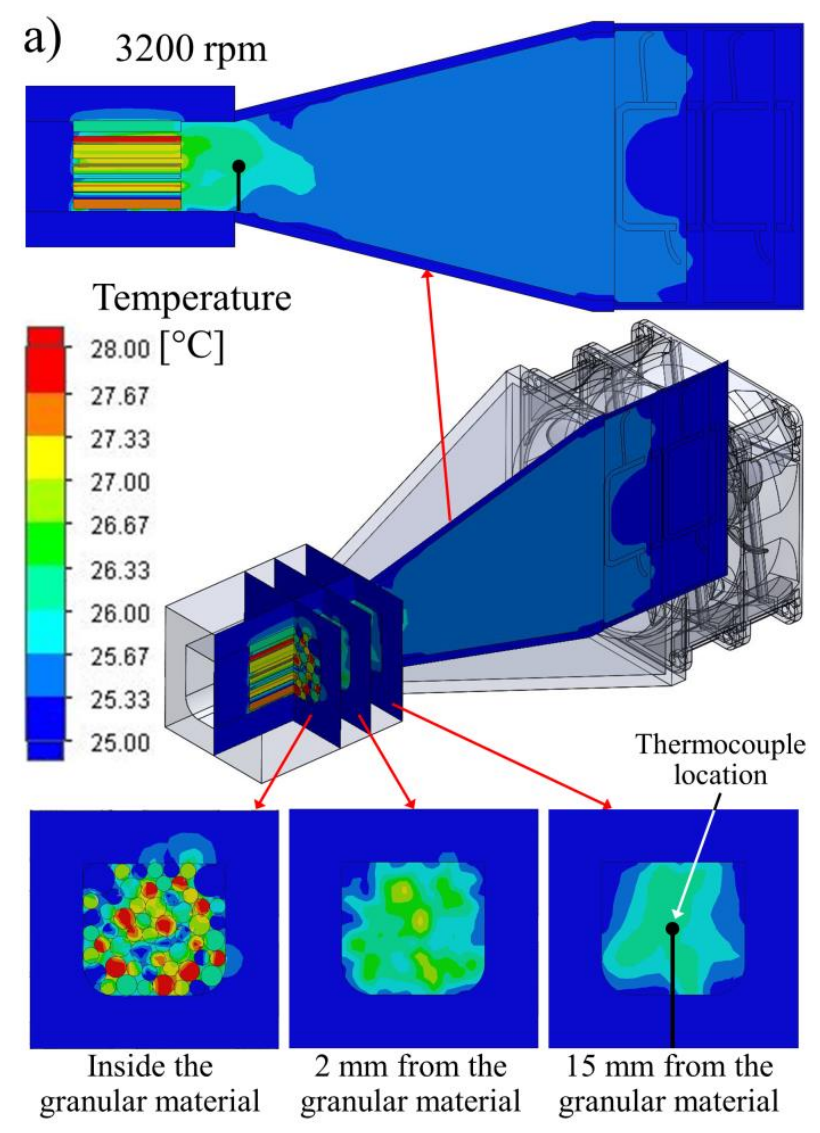

b)

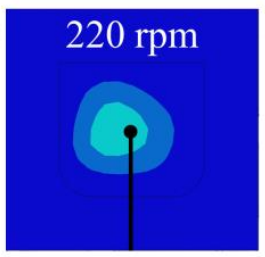

c) $1500 \mathrm{rpm}$

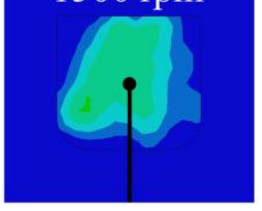

Fig. 10 Simulation results of air flow and heat transfer. 


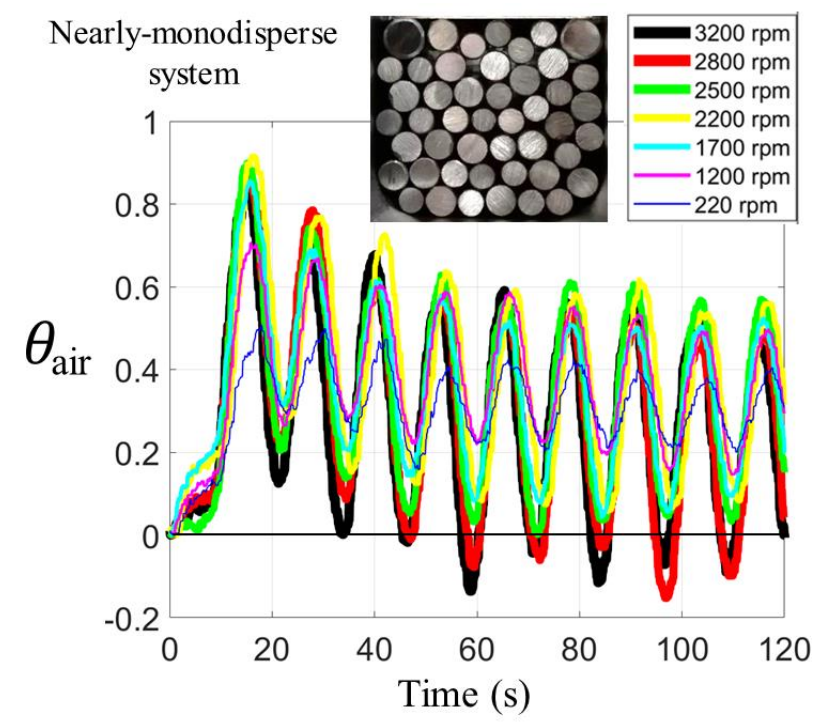

Fig. 11 Time variation in air temperature change $\theta_{\text {air }}$ for the nearly-monodisperse granular system subjected to cyclic confined compression. Fixed parameters: maximum vertical force $F_{\max }=-180 \mathrm{kN}$; force ratio $R=0.1$; loading frequency $f_{\mathrm{L}}=0.083 \mathrm{~Hz}$. Various fan rotation speeds, expressed in revolutions per minute (rpm), were compared. 


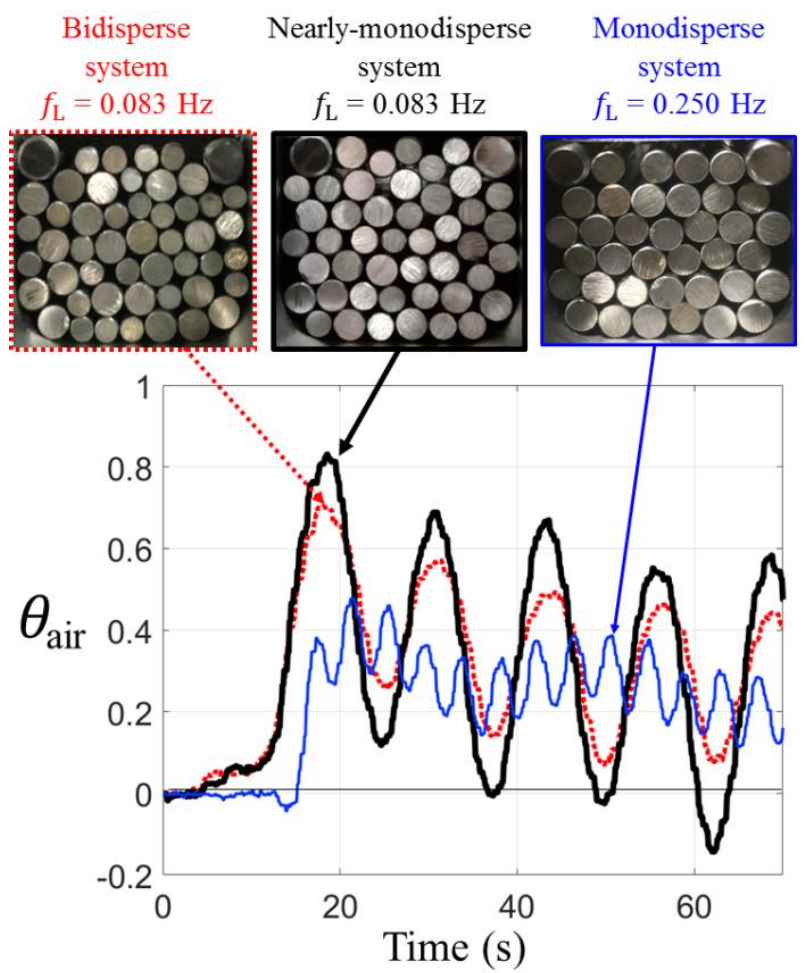

Fig. 12. Time variation in air temperature change $\theta_{\text {air }}$ for the three granular systems subjected to cyclic confined compression. Fixed parameters: maximum vertical force $F_{\max }=-180 \mathrm{kN}$; force ratio $R$ $=0.1 ;$ fan speed rotation $=3200$ revolutions per minute . 